\title{
Learning Curve Issues in Enterprise Application Integration Projects
}

\author{
Raffaella De Falco, Vincenzo Fabio Rollo, Gabriele Venturi* \\ *RCOST - University of Sannio - Benevento (ITALY) \\ defalco,rollo,venturi@unisannio.it
}

\begin{abstract}
Enterprise Application Integration (EAI) allows integration of existing legacy systems, packaged software (as ERP systems), and new developed systems. Starting from a previously proposed idea, in this paper the authors address the problem to collect input data for the model. The problem is approached using the Klenow's model, that aims at evaluating the efficiency improvement capability in using a technology. Here is carried on the analysis of a typical EAI architecture with the Goal Question Metrics (GQM) paradigm for the individualization of the questions. Appling the first two steps of GQM the authors obtained a list of question whose answer can help to assess the degree of adequacy of an EAI solution and the extent on which it can benefit from the learning curve.
\end{abstract}

\section{Introduction}

Enterprise Application Integration (EAI) is a very hot topic in corporate information systems. EAI provides for integrating several different systems in a coherent infrastructure. Therefore, it is often seen as an optimal solution to build a homogeneous environment for corporate intelligence and new services and products, while avoiding economical and organizational costs of system replacement.

EAI projects are often costly and risky, hence they undergo complex evaluation procedures and important decisions regarding them are taken at management levels far from the operational one. In reference [2] we analyzed the approaches commonly used to evaluate EAI solutions and showed that these are almost based on scope or scale economies models, at least for the assessment of direct costs and benefits of the alternatives. This is not surprising, because it happens for many business decisions. Nevertheless, we outlined two drawbacks that are the starting points for this work.
1. Economies of scale and scope models take into account some important factors, they but do not show the complete picture;

2. Current evaluation approaches can align the decisions to short term economical needs, but they risk hampering the capability to fulfill important strategic needs.

We have proposed a third model based on the economies of learning to better align decision procedures to strategic issues [2].

While it is not easy to link elements of the economies of learning models to the characteristics of an EAI project, it is relatively easy to collect data that feed the other models.

In this paper, after a brief overview of Enterprise Information System company strategic needs and the fitness of the outcomes of the economies of learning model to them, we focus on the problem of collecting input data for the model. We analyze the anatomy of an EAI project and apply the first two steps of the Goal Question Metric approach [1] to the individual activities, in order to establish what is important to know to evaluate an EAI solution with the proposed model.

\section{Strategic issues and EAI projects}

Integration activities have been carried on since the advent of company wide information systems. Their purposes and their technological and organizational characteristics evolved with the evolution of business.

The current business scenario can be characterized as a level 4 of the Courtney et al. [3] scale of uncertainty [2]. In a level 4 scenario there are two main issues for success:

1. Look for a high degree of leverage: try alternatives paying the lowest entry costs;

2. Don't get locked into one position by neglect: options and choices must be revaluated at least every six months.

This has an immediate impact on EAI, since the company success depends on the ability to develop 
new products and services, easily and rapidly (both for external or internal users), and/or new solutions for uphill integration $[6,8]$, knowing that only about $20 \%$ of them will survive the next year but that some of them, not knowing which, will develop a very large amount of transactions. This kind of activities is very different from traditional EAI activities. Traditional EAI projects are big and entail a long analysis phase and a step by step implementation. On the contrary, integration in the current competitive scenario is required to show the following characteristics:

A reduced project dimension: each project often aims to implement only a particular product or service;

A very short time to market: from a few weeks to a few months, rather than the years required for traditional integration project;

High requirements instability: Requirements can, and will, change rapidly during the development;

High frequency: These are repeated very often, possibly several times during the year, as the needs for new products and services emerge. However, even today integration must be conducted on a relatively stable platform composed of the company legacy system and of the EAI platform or framework in use. Since these activities are focused, frequently repeated, and require high degree of adaptability from both the systems and the people involved, these seem to be an ideal application field for learning theories.

\section{The Economies of Learning}

Modern economy is often recognized to be a learning economy, where change is rapid and old skills quickly become obsolete, therefore new skills are constantly required and organizations have no alternatives but to continuously update their technologies and retrain their workers [5]. Besides macroeconomic, managerial and social issues, the economies of learning approach is strictly tied to an older and more specific model. This model has been conceived in fundamental economical sectors like manufacturing with the aim of explaining why some production plants where showing a reduction of unitary costs overtime not explained by the two alternative models (scale and scope). Several empirical studies of plant productivity show that, when firms change their production technology, their productivity initially drops and then gradually rises to eventually overtake the level achieved with the old technology [4], as shown in figure 1.

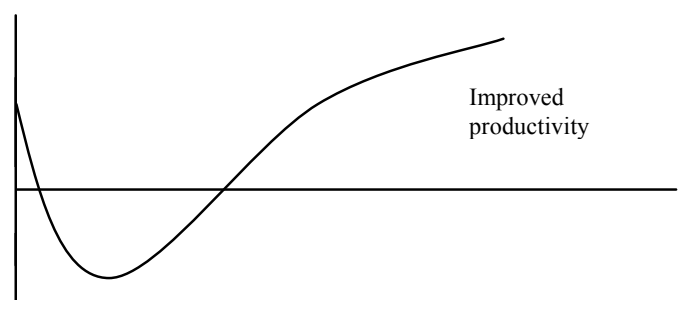

Knowledge Adjustment

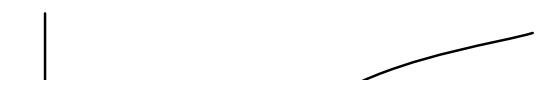

Figure 1. Productivity over time with learning effect

- injitiat fatling/productivity indicates that the new technolkgy use suffers from a lack of knowledge, , ile thon the people learn a lot auvu Knowledge Adjustment ation and swiftly become capable of reaching a higher level of productivity. The model implies that:

1. The productivity falls when a new technology is adopted, but grows quickly as the people acquire experience with the new technology.

2. It is easier to reap more rewards updating technology when the demand is high because an high rate of production pushes the workers to learn more quickly about the new technology.

3. There is a low correlation between productivity and labor input because the labor input is kept high using a new technology of which there is much to learn.

\section{Learning and EAI}

The Klenow model [4] is of a particular interest for the EAI topic because it explicitly takes into account the people learning as an important productivity gauging factor while the more traditional models, scale and scope, only consider the amount of use of a technology.

Using scale and scope factors for evaluating EAI infrastructure expected benefits means to take into account only parameters like the use of the solution among branches and the whole amount of transactions or processes using it. Although these are important issues, none of them has a direct impact on the strategic needs previously outlined, while the learning model can take these needs into account. On the other hand, there are several difficulties in applying the learning model:

1. It is not clear how to forecast the frequency of new services and products implementation;

2. It is not obvious which factors in an EAI project organization can boost or retain the learning;

3. It lacks an approach to collecting information on EAI project alternatives and transforming them in figures suitable to be used in a decisional process.

A common, very high level, architecture for EAI is shown in Fig.2. In it legacy systems are integrated by 
means of wrappers and adapters. The intermediate layer reconstructs, possibly in an object oriented fashion, the business workflows onto which it is possible to produce new services oriented both internally and externally to the company.

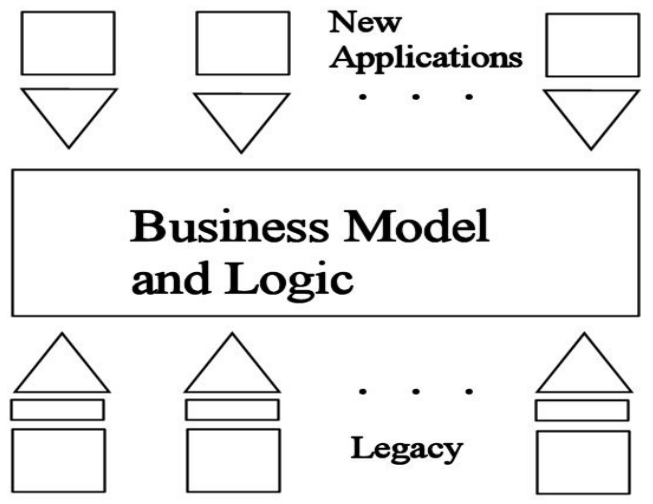

Figure 2. A more advanced architecture for EAI solutions.

In this architecture it is possible to recognise three main levels. From bottom to top: a first level in which the legacy systems are integrated in the solution, a second level that produces the integration using the legacy systems and other resources, and a third level in which new services are implemented. These levels are very different from each other with regard to their purposes, underlying technologies, and implications for the application of the economies of learning (EoL) paradigm as well. In the following three subsections we apply the GQM paradigm to the three levels.

The possible benefits stemming from learning theories application to EAI can come in the use phase, when a quite stable infrastructure is used to implement new products and services. Our aim is to link these possible benefits to characteristics of the EAI architecture and to the EAI implementation and startup phase as well. Therefore, in the following discussion, we put ourselves at the end of the startup phase in order to try to assess how the decision and first implementation phases impact on the EAI exploitation possibilities.

Since companies must obtain strategic benefits while competing in though markets, these must be careful when investing on possible future advantages.

We can summarize the principal risks and benefits of our approach with respect to more traditional ones in the following way:

\begin{tabular}{|l|l|}
\hline $\begin{array}{l}\text { Benefits: } \\
\text { The approach leads to largely } \\
\text { standard solutions, therefore it is easy } \\
\text { to outsource both implementation and } \\
\text { maintenance. }\end{array}$ & $\begin{array}{l}\text { Benefits: } \\
\text { It seems very fit to strategic needs: } \\
\text { It can lead to progressively reducing } \\
\text { costs for new product and services. }\end{array}$ \\
$\begin{array}{l}\text { The evaluation approach is well } \\
\text { known: accounting and management } \\
\text { are at ease with it. }\end{array}$ & \\
\hline $\begin{array}{l}\text { Drawbacks: } \\
\text { The cost for single future solution is, } \\
\text { roughly, function of the solution size. } \\
\text { The integration is difficult to become } \\
\text { a success factor because competitors } \\
\text { can acquire quite the same solution. }\end{array}$ & $\begin{array}{l}\text { Drawbacks: } \\
\text { It can be difficult to understand } \\
\text { integration project we can better } \\
\text { exploit learning. } \\
\text { It is not clear what data must be } \\
\text { collected to evaluate possible } \\
\text { learning benefits. } \\
\text { Accounting and management are not } \\
\text { accustomed to the approach. }\end{array}$ \\
\hline
\end{tabular}

Table 1. Benefits and drawbacks of the different approaches.

In Table 1 benefits and drawbacks of traditional approaches and the learning economy one are compared.

One of the drawbacks that we individuate for the learning approach is the lack of knowledge of learning theories by accountants and managers. Nevertheless, we believe that accountant and managers can readily become quite expert in using the new approach if it became relevant for the business. A related issues is the real interest of managers to take middle and long term approaches. This point is a very central one in current management concerns but is very far from the purposes of this work.

In the following we focus on issues related to the first two drawbacks of learning based approaches:

1. What data we need to evaluate the capability of a EAI infrastructure, both technological and organizational, to promote learning.

2. In a EAI project how we can point out the spots in which learning can lead to important benefits.

\subsection{EoL at the Legacy Level}

The business goals singled out for the legacy level are:

1. Not to disturb the present business processes based on the system with unforeseeable work loads generated by the integration.

2. To promptly react to new demands from the integrated level such as:

a) availability of data or services already on the system,

b) request to change something exposed by the system.

Type a) requests only impact the legacy administrators, while type b) requests can impact on the administrators and the users as well. \begin{tabular}{|l|l}
\hline Traditional approaches & Learning economy \\
\hline
\end{tabular} 
Considering the more complete case, where the legacy administrators are responsible for the wrappers and adapters, the questions related to the goals are:

1. Are the legacy administrators able to understand quickly and exactly what is required?

2. Do the administrators know the needs of the EAI platform?

3. If we consider the learning curve in relation to the technologies used for wrappers and adapters, at which point of this curve are administrators?

4. Is there a means to partially decouple the legacy systems from the EAI workloads or alternatively a clear comprehension of these by the legacy administrators?

5. If the change has any impact on the procedure used by the legacy users, are they supported in accepting the change?

In order to assess the learning curve exploitation by the legacy administrators, possible questions are:

1. What is the level of pre-existing knowledge by the legacy administrators of the used technologies in wrappers and adapters?

2. What is the level of involvement of the legacy staff in the integration project?

\subsection{EoL at the Business Model Level}

Goals for the integration level are:

1. To implement the communication among the systems and to support business intelligence;

2. To allow for a quickly implementation of the new product and services of the uphill layer (possibly implementing a model coherent with the business);

3. To allow the evolution of the implemented model in accordance with the business. That is, to be responsive to change requests coming from the top layer of the architecture;

Answers to the following questions can indicate the degree of fulfillment of these goals:

1. Is there in some workflow a labor intensive transfer of data?

2. Are the information needs of the company decision makers satisfied?

3. What is the overlapping degree between the set of the abstractions used talking about business and the set of the abstractions represented on the system?

4. Are there or have there been recent changes in the way of representing the business which are not represented in my model?

5. How many changes or innovations carried out at the vertical level impact in the integration level?

\subsection{EoL at the service and application Level}

The new services and applications level is the layer of the whole integrated system that directly must meet the outlined strategic needs. Therefore the goals for this level can be easily singled out.

1. Fast setup of new products and services.

2. Increasing efficiency in setting up new products and services.

The degree of fulfillment of these goals can be assessed with the following questions:

1. Are there requests for new products or services that are not satisfied?

2. Is there an overtime increase in efficiency in setting up new products and services? (Especially one that is explainable by the Klenow model?)

\section{Conclusions and future works}

In this paper we approached the problem to use a model, namely the Klenow's one, for the evaluation of EAI solutions. The use of such a model is very promising since it aims at evaluating the efficiency improvement capability in using a technology. This can be a very important point because it is obviously and directly tied to the strategic issue addressed by EAI projects. Here we carried on the analysis of a typical EAI architecture with the GQM paradigm until the individuation of the questions. Appling the first two steps of GQM we obtained a list of question whose answer can help to assess the degree of adequacy of an EAI solution and the extent on which it can benefit from the learning curve. Besides the obvious but not simple further step to find measures answering the questions, it is worth noticing that there is an important point regarding the whole integrated system. Two others very important questions are:

1. What is the rate at which requests of new services, or change of existing ones, in the top layer implies that changes must be made on the downhill ones?

2. Is this rate homogeneous for all the infrastructure or there are 'sensible' spots?

Knowing that can be really important to assess the relative importance of learning benefits for each layer and for each part of the different layers.

We consider our analysis can be carried on subdividing our research field on a vertical dimension and on a temporal one. The vertical levels are these previously individuated while the horizontal ones are the life phases of the integration. 
Our purpose is to take in account benefits that will be relevant in the use phase linking them to decisions taken in the previous two phases. Therefore the temporal scale allow us to consider these links.

Currently we are continuing on our work in three separated but connected directions:

1. For each layer we are working on measures that can be used to answer to the specific questions. It is obviously important to find a group of measures easy to take during the start up, and a group of factors that can possibly be measured on project during the decisions phase. The latter can be used to compare different alternatives and the first to monitor the project to be certain of reaching the planned benefits.

2. In the meantime we are addressing the problem to establish how to measure the degree of interconnection of the layers of an EAI project. This will help us to comprehend when the Klenow model should be applied to other layers beside the upper one and to what extent.

3. Another important research direction is about staffing and composition of teams working in setting up the EAI solution in relation to teams using it when operational. In this research we aim to comprehend how the knowledge about the implemented solutions can be retained and accumulated and what kinds of knowledge are more important to be taken into consideration .

\section{References}

[1] V.R. Basili, D.M. Weiss, "A Methodology for Collecting Valid Software Engineering Data" IEEE Transactions on Software Engineering, vol. SE-10, no.6, November 1984 pp.728-738.

[2] G.Canfora, V.F.Rollo, G.Venturi, "Business Change Impacts on System Integration”. ICEIS(1) 2003: 28-38.

[3] H.G. Courtney, J.Kirkland, and S.P.Viguerie, "Strategy Under Uncertainity"; Harvard Business Review, NovemberDecember 1997.

[4] P. Klenow, " Learning Curves and the Cyclical Behavior of Manufacturing Industries", Review of Economic Dynamics, 1998, vol. 1, issue 2, pages 531-550.

[5]B. Lundvall B.-A., "Competition and trasformation in the learning economy- illustrated by Danish case", DRUID Working Paper, September 1998,Department of Business Studies, Aalborg University.
[6] T. Puschmann, R. Alt, "Enterprise Application Integration: The Case of the Robert Bosch Group", Proceedings of the $34^{\text {th }}$ Hawaii International Conference in System Science, IEEE, 2001.

[7] W. Ruh, F. Maginnis, W. Brown, 'Enterprise Application Integration: A Wiley Tech Brief'; Wiley John Wiley \& Sons Inc., New York, USA, 2000

[8] M.Themistocleous, Z. Irani, "Evaluating and Adopting Application Integration: The case of a Multinational Petroleoum Company", Proceedings of the $35^{\text {th }}$ HICSE (Hawaii International Conference on System Science, IEEE, 2002. 